\title{
FURTHER CALCULATIONS FOR THE MCKEAN STOCHASTIC GAME FOR A SPECTRALLY NEGATIVE LÉVY PROCESS: FROM A POINT TO AN INTERVAL
}

\author{
E. J. BAURDOUX, ${ }^{*}$ London School of Economics \\ K. VAN SCHAIK, ${ }^{* *}$ University of Bath
}

\begin{abstract}
Following Baurdoux and Kyprianou (2008) we consider the McKean stochastic game, a game version of the McKean optimal stopping problem (American put), driven by a spectrally negative Lévy process. We improve their characterisation of a saddle point for this game when the driving process has a Gaussian component and negative jumps. In particular, we show that the exercise region of the minimiser consists of a singleton when the penalty parameter is larger than some threshold and 'thickens' to a full interval when the penalty parameter drops below this threshold. Expressions in terms of scale functions for the general case and in terms of polynomials for a specific jump diffusion case are provided.
\end{abstract}

Keywords: Stochastic game; optimal stopping; Lévy process; fluctuation theory

2010 Mathematics Subject Classification: Primary 60G40; 91A15

\section{Introduction}

This paper is a follow-up to the paper [2] by Baurdoux and Kyprianou (henceforth BK), in which the solution to the McKean stochastic game driven by a spectrally negative Lévy process is studied. Let us introduce the setting in BK (and in this paper). Let $X$ be a Lévy process defined on a filtered probability space $(\Omega, \mathcal{F}, \mathbb{F}, \mathrm{P})$, where $\mathbb{F}=\left(\mathcal{F}_{t}\right)_{t \geq 0}$ is the filtration generated by $X$ which is naturally enlarged (cf. Definition 1.3 .38 of [6]). For $x \in \mathbb{R}$, we denote by $\mathrm{P}_{x}$ the law of $X$ when it is started at $x$ and we abbreviate $\mathrm{P}=\mathrm{P}_{0}$. Accordingly, we shall write $\mathrm{E}_{x}$ and $\mathrm{E}$ for the associated expectation operators. We assume throughout that $X$ is spectrally negative, meaning that it has no positive jumps and that it is not the negative of a subordinator.

The McKean stochastic game is an example of a type of stochastic game introduced by Dynkin [9]. It is a two-player zero-sum game, consisting of a maximiser aiming at maximising over $\mathbb{F}$-stopping times $\tau$ the expected payoff according to the (discounted) lower payoff process given by $\mathrm{e}^{-q t}\left(K-\exp \left(X_{t}\right)\right)^{+}$for all $t \geq 0$, and a minimiser aiming at minimising over F-stopping times $\sigma$ the expected payoff according to the (discounted) upper payoff process given by $\mathrm{e}^{-q t}\left(\left(K-\exp \left(X_{t}\right)\right)^{+}+\delta\right)$ for all $t \geq 0$, where $K, \delta>0$. That is, for any pair of stopping times $(\tau, \sigma)$, the payoff to the maximiser is

$$
M_{x}(\tau, \sigma):=\mathrm{E}_{x}\left[\mathrm{e}^{-q \tau}\left(K-\mathrm{e}^{X_{\tau}}\right)^{+} \mathbf{1}_{\{\tau \leq \sigma\}}+\mathrm{e}^{-q \sigma}\left(\left(K-\mathrm{e}^{X_{\sigma}}\right)^{+}+\delta\right) \mathbf{1}_{\{\sigma<\tau\}}\right] .
$$

Received 2 November 2009; revision received 15 November 2010.

* Postal address: Department of Statistics, London School of Economics, Houghton Street, London, WC2A 2AE, UK. Email address: e.j.baurdoux@1se.ac.uk

** Current address: School of Mathematics, University of Manchester, Oxford Road, Manchester, M13 9PL, UK.

Email address: kees.vanschaik@manchester.ac.uk

This author gratefully acknowledges being supported by a postdoctoral grant from the AXA Research Fund. 
We assume throughout this paper that the discount factor $q$ satisfies

$$
0 \leq \psi(1) \leq q \text { and } q>0
$$

where $\psi$ denotes the Laplace exponent of $X$. For a spectrally negative Lévy process $X$, this Laplace exponent is of the form

$$
\psi(\lambda)=a \lambda+\frac{1}{2} \sigma_{X}^{2} \lambda^{2}+\int_{(-\infty, 0)}\left(\mathrm{e}^{\lambda x}-1-\lambda x \mathbf{1}_{\{x>-1\}}\right) \Pi(\mathrm{d} x)
$$

for $\lambda \geq 0$. Here $\sigma_{X}$ is called the Gaussian coefficient of $X$ and $\Pi$ is the Lévy measure which satisfies $\int_{(-\infty, 0)}\left(1 \wedge x^{2}\right) \Pi(\mathrm{d} x)<\infty$.

Note that, since both payoff processes vanish almost surely as $t \rightarrow \infty$, there is no ambiguity in allowing for $\tau$ and $\sigma$ to be infinitely valued, as we do in this paper. For any $x$, this game has a value if the upper and lower values, $\inf _{\sigma} \sup _{\tau} M_{x}(\tau, \sigma)$ and $\sup _{\tau} \inf _{\sigma} M_{x}(\tau, \sigma)$, respectively, coincide. Furthermore, if a pair $\left(\tau^{*}, \sigma^{*}\right)$ exists such that

$$
M_{x}\left(\tau, \sigma^{*}\right) \leq M_{x}\left(\tau^{*}, \sigma^{*}\right) \leq M_{x}\left(\tau^{*}, \sigma\right) \text { for all }(\tau, \sigma),
$$

the value exists and equals $M_{x}\left(\tau^{*}, \sigma^{*}\right)$. In this case $\left(\tau^{*}, \sigma^{*}\right)$ is called a saddle point (or Nash equilibrium). For an account of these concepts in a general Markovian setting, see [10] and the references therein. For other examples of stochastic games, see, e.g. [3], [4], [11], [13], and [15].

Note that the McKean game can be seen as an extension of the classic McKean optimal stopping problem (cf. [17] and Theorem 1 below). In a financial interpretation, this optimal stopping problem is usually referred to as an American put option, with $K$ the strike price. The McKean game then extends the American put option by introducing the possibility for the writer of the option to cancel the contract, at the expense of paying the intrinsic value plus an extra constant penalty given by the penalty parameter $\delta$. Cf., e.g. [12] and [13] for a general account on the interpretation of stochastic games as financial contracts.

In BK it was shown that a saddle point $\left(\tau^{*}, \sigma^{*}\right)$ indeed exists for the McKean game, so, in particular, the value function $V$ is well defined by

$$
\begin{aligned}
V(x) & =\sup _{\tau} \inf _{\sigma} \mathrm{E}_{x}\left[\mathrm{e}^{-q \tau}\left(K-\mathrm{e}^{X_{\tau}}\right)^{+} \mathbf{1}_{\{\tau \leq \sigma\}}+\mathrm{e}^{-q \sigma}\left(\left(K-\mathrm{e}^{X_{\sigma}}\right)^{+}+\delta\right) \mathbf{1}_{\{\sigma<\tau\}}\right] \\
& =\inf _{\sigma} \sup _{\tau} \mathrm{E}_{x}\left[\mathrm{e}^{-q \tau}\left(K-\mathrm{e}^{X_{\tau}}\right)^{+} \mathbf{1}_{\{\tau \leq \sigma\}}+\mathrm{e}^{-q \sigma}\left(\left(K-\mathrm{e}^{X_{\sigma}}\right)^{+}+\delta\right) \mathbf{1}_{\{\sigma<\tau\}}\right] \\
& =\mathrm{E}_{x}\left[\mathrm{e}^{-q \tau^{*}}\left(K-\mathrm{e}^{X_{\tau^{*}}}\right)^{+} \mathbf{1}_{\left\{\tau^{*} \leq \sigma^{*}\right\}}+\mathrm{e}^{-q \sigma^{*}}\left(\left(K-\mathrm{e}^{X_{\sigma^{*}}}\right)^{+}+\delta\right) \mathbf{1}_{\left\{\sigma^{*}<\tau^{*}\right\}}\right] .
\end{aligned}
$$

The optimal stopping time for the maximiser, $\tau^{*}$, is the first hitting time of an interval of the form $\left(-\infty, x^{*}\right]$ for some $x^{*}<\log K$. For the minimiser, the optimal stopping time $\sigma^{*}$ is as follows. When the penalty parameter $\delta$ exceeds $\bar{\delta}:=U(\log K)$, where $U$ denotes the value function of the McKean optimal stopping problem, the minimiser never stops (i.e. $\sigma^{*}=\infty$ ). When $\delta<\bar{\delta}$, the optimal stopping region for the minimiser is an interval of the form [ $\left.\log K, y^{*}\right]$. If the Gaussian component $\sigma_{X}$ of $X$ is equal to 0 (note that this corresponds to the situation that $X$ does not creep downwards), we have $y^{*}>\log K$. Furthermore, formulae in terms of scale functions for $x^{*}$ and $V$ on $(-\infty, \log K]$ were provided.

Two issues were however left open in BK. Firstly, when $X$ has a Gaussian component, it was not clear when the optimal stopping region for the minimiser consists of a point and when of 
an interval, i.e. when $y^{*}=\log K$ and when $y^{*}>\log K$ holds. Secondly, no characterisation was given of $y^{*}$. In this paper we give answers to both these issues. In particular, we show that, when $\sigma_{X}>0$, there exists a critical value $\delta_{0} \in(0, \bar{\delta})$ such that the stopping region for the minimiser is a single point when $\delta \in\left[\delta_{0}, \bar{\delta}\right)$ and a full interval when $\delta \in\left(0, \delta_{0}\right)$; cf. Theorem 4 (see also Remark 1). Furthermore, we show that $y^{*}$ and $\delta_{0}$ can be characterised as unique solutions to functional equations using scale functions; cf. Theorem 5 .

The rest of this paper is organised as follows. In the remainder of this introduction we introduce scale functions and some notation (Subsection 1.1), and review the results from BK in more detail (Subsection 1.2). In Section 2 we present our new results. Finally, in Section 3 we translate these results to a specific jump diffusion setting, accompanied by some plots.

\subsection{Scale functions}

First we introduce some notation for first entry times. For $a \leq b$, we write

$$
\begin{gathered}
\tau_{a}^{+}:=\inf \left\{t>0 \mid X_{t}>a\right\}, \quad \tau_{a}^{-}:=\inf \left\{t>0 \mid X_{t}<a\right\} \\
\text { and } T_{[a, b]}:=\inf \left\{t>0 \mid X_{t} \in[a, b]\right\} .
\end{gathered}
$$

Furthermore, we denote the often used first hitting time of $\log K$ for simplicity by $T_{K}$, that is, $T_{K}:=\inf \left\{t>0 \mid X_{t}=\log K\right\}$.

A useful class of functions when studying first exit problems driven by spectrally negative Lévy processes are so-called scale functions. We shortly review some of their properties as they play an important role in this paper; for a more complete overview, the reader is, e.g. referred to Chapter VII of [5] or Chapter 8 of [16]. For each $q \geq 0$, the scale functions $W^{(q)}: \mathbb{R} \rightarrow[0, \infty)$ are known to satisfy, for all $x \in \mathbb{R}$ and $a \geq 0$,

$$
\mathrm{E}_{\mathrm{x}}\left[\mathrm{e}^{-q \tau_{a}^{+}} \mathbf{1}_{\left\{\tau_{a}^{+}<\tau_{0}^{-}\right\}}\right]=\frac{W^{(q)}(x \wedge a)}{W^{(q)}(a)} .
$$

In particular, it is evident that $W^{(q)}(x)=0$ for all $x<0$. Furthermore, it is known that $W^{(q)}$ is almost everywhere differentiable on $(0, \infty)$, it is right continuous at 0 , and

$$
\int_{0}^{\infty} \mathrm{e}^{-\beta x} W^{(q)}(x) \mathrm{d} x=\frac{1}{\psi(\beta)-q}
$$

for all $\beta>\Phi(q)$, where $\Phi(q)$ is the largest root of the equation $\psi(\theta)=q$ (of which there are at most two-recall that $\psi$ is the Laplace exponent of $X$ ). We shall assume throughout this paper that the jump measure $\Pi$ has no atoms when $X$ is of bounded variation, which implies that $W^{(q)} \in C^{1}(0, \infty)$ (see [8]). In the case when $X$ has Gaussian component $\sigma_{X}>0$, it is known that $W^{(q)} \in C^{2}(0, \infty)$ with $W^{(q)}(0)=0$ and $W^{(q) \prime}(0)=2 / \sigma_{X}^{2}$. We usually write $W=W^{(0)}$.

Associated to the functions $W^{(q)}$ are the functions $Z^{(q)}: \mathbb{R} \rightarrow[1, \infty)$ defined by

$$
Z^{(q)}(x)=1+q \int_{0}^{x} W^{(q)}(y) \mathrm{d} y
$$

for $q \geq 0$. Together, the functions $W^{(q)}$ and $Z^{(q)}$ are collectively known as scale functions and predominantly appear in almost all fluctuation identities for spectrally negative Lévy processes. For example, it is also known that, for all $x \in \mathbb{R}$ and $a, q \geq 0$,

$$
\mathrm{E}_{x}\left[\mathrm{e}^{-q \tau_{0}^{-}} \mathbf{1}_{\left\{\tau_{a}^{+}>\tau_{0}^{-}\right\}}\right]=Z^{(q)}(x \wedge a)-\frac{Z^{(q)}(a)}{W^{(q)}(a)} W^{(q)}(x \wedge a)
$$


and

$$
\mathrm{E}_{x}\left[\mathrm{e}^{-q \tau_{0}^{-}} \mathbf{1}_{\left\{\tau_{0}^{-}<\infty\right\}}\right]=Z^{(q)}(x)-\frac{q}{\Phi(q)} W^{(q)}(x),
$$

where $q / \Phi(q)$ is to be understood in the limiting sense $\psi^{\prime}(0) \wedge 0$ when $q=0$.

For $c>0$, consider the change of measure

$$
\left.\frac{\mathrm{dP}^{c}}{\mathrm{dP}}\right|_{\mathcal{F}_{t}}=\mathrm{e}^{c X_{t}-\psi(c) t} .
$$

Under $\mathrm{P}^{c}$, the process $X$ is still a spectrally negative Lévy process, and we mark its Laplace exponent and scale functions with the subscript $c$. From $\psi_{c}(\lambda)=\psi(\lambda+c)-\psi(c)$ for $\lambda \geq 0$ we get, by taking Laplace transforms,

$$
W_{c}^{(q)}(x)=\mathrm{e}^{-c x} W^{(q+\psi(c))}(x)
$$

for all $q \geq 0$ and, similarly,

$$
Z_{c}^{(q)}(x)=1+q \int_{0}^{x} W_{c}^{(q)}(y) \mathrm{d} y .
$$

\subsection{Reviewing the McKean stochastic game}

First consider the McKean optimal stopping problem (or American put option) with value function $U$, i.e.

$$
U(x)=\sup _{\tau} \mathrm{E}_{x}\left[\mathrm{e}^{-q \tau}\left(K-\mathrm{e}^{X_{\tau}}\right)^{+}\right] .
$$

We recall the solution to this problem as it appears in [7] (see also [18]).

Theorem 1. For the McKean optimal stopping problem, under (1), we have

$$
U(x)=K Z^{(q)}\left(x-k^{*}\right)-\mathrm{e}^{x} Z_{1}^{(q-\psi(1))}\left(x-k^{*}\right),
$$

where

$$
\mathrm{e}^{k^{*}}=K \frac{q}{\Phi(q)} \frac{\Phi(q)-1}{q-\psi(1)},
$$

which is to be understood in the limiting sense when $q=\psi(1)$, i.e. $\mathrm{e}^{k^{*}}=K \psi(1) / \psi^{\prime}(1)$. An optimal stopping time is given by $\tau^{*}=\inf \left\{t>0: X_{t}<k^{*}\right\}$.

Next we recall the main result from BK on a saddle point and the value function for the McKean game.

Theorem 2. Consider the McKean stochastic game under assumption (1) and recall that $\bar{\delta}=U(\log K)$.

(i) If $\delta \geq \bar{\delta}$ then a stochastic saddle point is given by $\tau^{*}$ from Theorem 1 and $\sigma^{*}=\infty$, in which case $V=U$.

(ii) If $\delta<\bar{\delta}$, a stochastic saddle point is given by the pair

$$
\tau^{*}=\inf \left\{t>0: X_{t}<x^{*}\right\} \text { and } \sigma^{*}=\inf \left\{t>0: X_{t} \in\left[\log K, y^{*}\right]\right\},
$$

where $x^{*}$ uniquely solves

$$
Z^{(q)}(\log K-x)-Z_{1}^{(q-\psi(1))}(\log K-x)=\frac{\delta}{K},
$$


$x^{*}>k^{*}$ (the optimal level of the corresponding McKean optimal stopping problem in Theorem 1), and $y^{*} \geq \log K$.

Furthermore,

$$
V(x)=K Z^{(q)}\left(x-x^{*}\right)-\mathrm{e}^{x} Z_{1}^{(q-\psi(1))}\left(x-x^{*}\right)
$$

for $x \leq \log K$ and if $y^{*}=\log K$ then, for any $x \in \mathbb{R}$,

$$
V(x)=K Z^{(q)}\left(x-x^{*}\right)-\mathrm{e}^{x} Z_{1}^{(q-\psi(1))}\left(x-x^{*}\right)+\alpha \mathrm{e}^{\Phi(q)\left(\log K-x^{*}\right)} W^{(q)}(x-\log K),
$$

where

$$
\alpha=\mathrm{e}^{x^{*}} \frac{q-\psi(1)}{\Phi(q)-1}-\frac{q K}{\Phi(q)},
$$

which is to be understood in the limiting sense when $q=\psi(1)$, i.e. $\alpha=\mathrm{e}^{x^{*}} \psi^{\prime}(1)-$ $K \psi(1)$.

Hence, a saddle point exists, and consists of the first hitting time of $\left(-\infty, x^{*}\right]$ for the maximiser and of the first hitting time of $\left[\log K, y^{*}\right]$ for the minimiser. Note that, when $\delta<\bar{\delta}$, we know that the value of the McKean is not everywhere equal to that of its oneplayer counterpart, as $V(\log K)=\delta<U(\log K)$ in that case. Furthermore, (6) gives us a characterisation of $x^{*}$, but we know only a little about $y^{*}$.

In BK, the issue of whether $y^{*}=\log K$ or $y^{*}>\log K$ was answered only when $X$ has no Gaussian component.

Theorem 3. Suppose in Theorem 2 that $\delta<\bar{\delta}$. If $X$ has no Gaussian component then $y^{*}>$ $\log K$ and necessarily $\Pi\left(-\infty, \log K-y^{*}\right)>0$.

Remark 1. We now discuss the intuitive interpretation of these results.

Owing to the fact that $X$ has no positive jumps, the choice of $y^{*}$ has no influence when $X$ is started at a value to the left of $\log K$ as the minimiser would stop as soon as the process hits $\log K$. However, the situation is different when starting from any $X_{0}>\log K$; the minimiser could either stop right away and pay $\delta$ to the maximiser, or wait a short time. From the minimiser's point of view, the latter decision has the advantage of profiting from the discounting, but the disadvantage of the risk that a (large) negative jump could bring $X$ (far) below $\log K$, where a higher payoff than (discounted) $\delta$ can be claimed by the maximiser. The closer $X_{0}$ is chosen to $\log K$, the more dominant the disadvantage becomes and, hence, the exercise region for the minimiser can take the form of an interval [log $\left.K, y^{*}\right]$.

When $X$ is a Brownian motion, it is obvious that we have $y^{*}=\log K$ for any $\delta \in(0, \bar{\delta}]$ (see also [15]), as then the process can only get below $\log K$ by hitting it first.

Theorem 3 tells us that the other extreme case, namely $y^{*}>\log K$ for any $\delta \in(0, \bar{\delta}]$, i.e. the disadvantage of waiting being dominant for the minimiser, occurs whenever $X$ has no Gaussian component.

The interesting question is, what happens when $X$ has a Gaussian component and negative jumps? Then namely there is a trade-off between the possibility of the process entering the region $(-\infty, \log K]$ continuously (which can occur only when $\sigma_{X}>0$ ), leading to a relatively small payoff, and the possibility of passing $\log K$ by a jump, potentially leading to a larger payoff. It turns out that, for large enough $\delta$, when stopping immediately is relatively expensive, the Gaussian part 'wins' in the sense that $y^{*}=\log K$ (the minimiser is happy to take the risk of the process jumping to a less favourable region), while, for small enough $\delta$, when stopping 
immediately has become cheaper, the negative jumps 'win' in the sense that $y^{*}>\log K$; see Theorem 4 below. In fact, in Figure 4 at the end of this paper we plot the negative relationship between $y^{*}$ and $\sigma_{X}$ in the jump diffusion case.

\section{Single point or interval when $X$ has Gaussian part $\sigma_{X}>0$}

Throughout this section, we assume that condition (1) holds. Recall that $T_{K}:=\inf \{t>$ $\left.0 \mid X_{t}=\log K\right\}$. Consider the function

$$
f_{\delta}(x)=\sup _{\tau} \mathrm{E}_{x}\left[\mathrm{e}^{-q \tau}\left(K-\mathrm{e}^{X_{\tau}}\right) \mathbf{1}_{\left\{\tau \leq T_{K}\right\}}+\delta \mathrm{e}^{-q T_{K}} \mathbf{1}_{\left\{T_{K}<\tau\right\}}\right],
$$

i.e. the optimal value for the maximiser provided that the minimiser only exercises when $X$ hits $\log K$.

We first prove the following technical result.

Lemma 1. Suppose that $\sigma_{X}>0$ and $0<\delta \leq \bar{\delta}=U(\log K)$. The function $f_{\delta}$ is differentiable on $\mathbb{R} \backslash\{\log K\}$. Furthermore, $f_{\delta}=V$ on $(-\infty, \log K], f_{\delta} \geq V$ on $\mathbb{R}$, and $f_{\delta}^{\prime}(\log K+)$ is a strictly decreasing continuous function of $\delta$.

Proof. Let $\delta \in(0, \bar{\delta}]$. Owing to Theorem 2 and the absence of positive jumps, we have, for $x \leq \log K$,

$$
\begin{aligned}
V(x) & =\mathrm{E}_{x}\left[\mathrm{e}^{-q \tau_{x^{*}(\delta)}^{-}}\left(K-\exp \left(X_{\tau_{x^{*}(\delta)}^{-}}\right)\right) \mathbf{1}_{\left\{\tau_{x^{*}(\delta)}^{-}<T_{K}\right\}}+\delta \mathrm{e}^{-q T_{K}} \mathbf{1}_{\left\{T_{K}<\tau_{x^{*}(\delta)}^{-}\right.}\right] \\
& =\sup _{\tau} \mathrm{E}_{x}\left[\mathrm{e}^{-q \tau}\left(K-\mathrm{e}^{X_{\tau}}\right) \mathbf{1}_{\left\{\tau<T_{K}\right\}}+\delta \mathrm{e}^{-q T_{K}} \mathbf{1}_{\left\{T_{K}<\tau\right\}}\right] \\
& =f_{\delta}(x) .
\end{aligned}
$$

Also, for any $x \in \mathbb{R}$,

$$
\begin{aligned}
f_{\delta}(x) & =\sup _{\tau} \mathrm{E}_{x}\left[\mathrm{e}^{-q \tau}\left(K-\mathrm{e}^{X_{\tau}}\right) \mathbf{1}_{\left\{\tau \leq T_{K}\right\}}+\delta \mathrm{e}^{-q T_{K}} \mathbf{1}_{\left\{T_{K}<\tau\right\}}\right] \\
& \geq \inf _{\sigma} \sup _{\tau} \mathrm{E}_{x}\left[\mathrm{e}^{-q \tau}\left(K-\mathrm{e}^{X_{\tau}}\right) \mathbf{1}_{\{\tau \leq \sigma\}}+\delta \mathrm{e}^{-q \sigma} \mathbf{1}_{\{\sigma<\tau\}}\right] \\
& =V(x) .
\end{aligned}
$$

In fact, since stopping is not optimal on $(\log K, \infty)$, as the lower payoff function is 0 there, we deduce that, for all $x \in \mathbb{R}$,

$$
f_{\delta}(x)=\mathrm{E}_{x}\left[\mathrm{e}^{-q \tau_{x^{*}(\delta)}^{-}}\left(K-\exp \left(X_{\tau_{x^{*}(\delta)}^{-}}\right)\right) \mathbf{1}_{\left\{\tau_{x^{*}(\delta)}^{-} \leq T_{K}\right\}}+\delta \mathrm{e}^{-q T_{K}} \mathbf{1}_{\left\{T_{K}<\tau_{x^{*}(\delta)}^{-}\right.}\right] .
$$

Now, let $\delta_{2}>\delta_{1}>c$ for some $c>0$. From the definition of $f_{\delta}$ in (8) we find that

$$
\begin{aligned}
f_{\delta_{2}}(x)-f_{\delta_{1}}(x)= & \sup _{\tau} \mathrm{E}_{x}\left[\mathrm{e}^{-q \tau}\left(K-\mathrm{e}^{X_{\tau}}\right) \mathbf{1}_{\left\{\tau \leq T_{K}\right\}}+\delta_{2} \mathrm{e}^{-q T_{K}} \mathbf{1}_{\left\{T_{K}<\tau\right\}}\right] \\
& -\sup _{\tau} \mathrm{E}_{x}\left[\mathrm{e}^{-q \tau}\left(K-\mathrm{e}^{X_{\tau}}\right) \mathbf{1}_{\left\{\tau \leq T_{K}\right\}}+\delta_{1} \mathrm{e}^{-q T_{K}} \mathbf{1}_{\left\{T_{K}<\tau\right\}}\right] \\
\leq & \left(\delta_{2}-\delta_{1}\right) \sup _{\tau} \mathrm{E}_{x}\left[\mathrm{e}^{-q T_{K}} \mathbf{1}_{\left\{T_{K}<\tau\right\}}\right] \\
\leq & \left(\delta_{2}-\delta_{1}\right) \mathrm{E}_{x}\left[\mathrm{e}^{-q \tau_{\log K}^{-}}\right],
\end{aligned}
$$


from which we obtain (where the equality follows by (4))

$$
\begin{aligned}
\frac{f_{\delta_{2}}(\log K+\varepsilon)-\delta_{2}}{\varepsilon}-\frac{f_{\delta_{1}}(\log K+\varepsilon)-\delta_{1}}{\varepsilon} & \leq\left(\delta_{2}-\delta_{1}\right) \frac{\mathrm{E}_{\log K+\varepsilon}\left[\mathrm{e}^{-q \tau_{\log K}^{-}}\right]-1}{\varepsilon} \\
& =\left(\delta_{2}-\delta_{1}\right)\left(\frac{Z^{(q)}(\varepsilon)-1}{\varepsilon}-\frac{q}{\Phi(q)} \frac{W^{(q)}(\varepsilon)}{\varepsilon}\right) .
\end{aligned}
$$

Since $f_{\delta}$ is a differentiable function on $[\log K, \infty)$ (see Equation (27) of BK together with (9)), and using $Z^{(q)^{\prime}}(0)=W^{(q)}(0)=0$ and $W^{(q) \prime}(0+)=2 / \sigma_{X}^{2}$, we deduce that

$$
f_{\delta_{2}}^{\prime}(\log K+)-f_{\delta_{1}}^{\prime}(\log K+) \leq-\frac{2 q}{\sigma_{X}^{2} \Phi(q)}\left(\delta_{2}-\delta_{1}\right),
$$

showing that $f_{\delta}^{\prime}(\log K+)$ is strictly decreasing in $\delta$.

Also, using (8) and the fact that $\tau_{x^{*}\left(\delta_{1}\right)}^{-}$is a feasible strategy also when $\delta=\delta_{2}$, it holds that

$$
\begin{aligned}
f_{\delta_{2}}(x)-f_{\delta_{1}}(x) \geq & \mathrm{E}_{x}\left[\mathrm{e}^{-q \tau_{x^{*}\left(\delta_{1}\right)}^{-}}\left(K-\exp \left(X_{\tau_{x^{*}\left(\delta_{1}\right)}^{-}}\right)\right) \mathbf{1}_{\left\{\tau_{x^{*}\left(\delta_{1}\right)}^{-} \leq T_{K}\right\}}+\delta_{2} \mathrm{e}^{-q T_{K}} \mathbf{1}_{\left\{T_{K}<\tau_{x^{*}\left(\delta_{1}\right)}^{-}\right\}}\right] \\
& -\mathrm{E}_{x}\left[\mathrm{e}^{-q \tau_{x^{*}\left(\delta_{1}\right)}^{-}}\left(K-\exp \left(X_{\tau_{x^{*}\left(\delta_{1}\right)}^{-}}\right)\right) \mathbf{1}_{\left\{\tau_{x^{*}\left(\delta_{1}\right)}^{-} \leq T_{K}\right\}}+\delta_{1} \mathrm{e}^{-q T_{K}} \mathbf{1}_{\left\{T_{K}<\tau_{x^{*}\left(\delta_{1}\right)}^{-}\right\}}\right] \\
= & \left(\delta_{2}-\delta_{1}\right) \mathrm{E}_{x}\left[\mathrm{e}^{-q T_{K}} \mathbf{1}_{\left\{T_{K}<\tau_{x^{*}\left(\delta_{1}\right)}^{-}\right\}}\right] \\
\geq & \left(\delta_{2}-\delta_{1}\right) \mathrm{E}_{x}\left[\mathrm{e}^{-q T_{K}} \mathbf{1}_{\left\{T_{K}<\tau_{x^{*}(c)}^{-}\right\}}\right],
\end{aligned}
$$

where the final inequality follows from the observation that $x^{*}(\delta)$ is decreasing in $\delta$ and that $\delta_{1}>c$. Note that $x^{*}(c)<\log (K-c)$ since $V(x)$ is strictly decreasing in $x \in(-\infty, \log K]$ for any $\delta>0$ and, thus,

$$
\begin{gathered}
\frac{f_{\delta_{2}}(\log K+\varepsilon)-\delta_{2}}{\varepsilon}-\frac{f_{\delta_{1}}(\log K+\varepsilon)-\delta_{1}}{\varepsilon} \\
\geq\left(\delta_{2}-\delta_{1}\right) \frac{\mathrm{E}_{\log K+\varepsilon}\left[\mathrm{e}^{-q T_{K}} \mathbf{1}_{\left\{T_{K}<\tau_{x^{*}(c)}^{-}\right\}}\right]-1}{\varepsilon} \\
=\left(\delta_{2}-\delta_{1}\right) \frac{W^{(q)}\left(\log K+\varepsilon-x^{*}(c)\right)-W^{(q)}\left(\log K-x^{*}(c)\right)}{\varepsilon W^{(q)}\left(\log K-x^{*}(c)\right)} \\
-\left(\delta_{2}-\delta_{1}\right) \mathrm{e}^{\Phi(q)\left(\log K-x^{*}(c)\right)} \frac{W^{(q)}(\varepsilon)}{\varepsilon W^{(q)}\left(\log K-x^{*}(c)\right)},
\end{gathered}
$$

by Lemma 12 of BK. It follows that

$$
f_{\delta_{1}}^{\prime}(\log K+)-f_{\delta_{1}}^{\prime}(\log K+) \geq\left(\delta_{2}-\delta_{1}\right) \frac{\sigma_{X}^{2} W^{(q) \prime}\left(\log K-x^{*}(c)\right)-2 \mathrm{e}^{\Phi(q)\left(\log K-x^{*}(c)\right)}}{\sigma_{X}^{2} W^{(q)}\left(\log K-x^{*}(c)\right)} .
$$

Since $c$ is arbitrary, we conclude from this inequality together with (10) that $f_{\delta}^{\prime}(\log K+)$ is indeed continuous in $\delta$ for any $\delta>0$.

Now we are ready to prove our main result, extending Theorem 2.

Theorem 4. Suppose that $\sigma_{X}>0$. When $\Pi \neq 0$, there exists a unique $\delta_{0} \in(0, \bar{\delta})$ such that an optimal stopping time for the minimiser is given by $T_{K}$ (i.e. $y^{*}(\delta)=\log K$ ) when $\delta \in\left[\delta_{0}, \bar{\delta}\right]$ and by $T_{\left[\log K, y^{*}(\delta)\right]}$ for some $y^{*}(\delta)>\log K$ when $\delta \in\left(0, \delta_{0}\right)$. 
Proof. Let $\sigma_{X}>0$, and suppose that $\Pi \neq 0$. We know from Theorem 2 that the stopping region for the minimiser is of the form $\left[\log K, y^{*}\right]$ for some $y^{*} \geq \log K$. We claim that setting $\delta_{0}$ equal to the unique 0 of $f_{\delta}^{\prime}(\log K+)$ on $(0, \bar{\delta})$ yields the result.

First let us show that this unique 0 does indeed exist. For $\delta=\bar{\delta}$, it holds that $f_{\delta}^{\prime}(\log K+)=$ $U^{\prime}(\log K)<0$ (cf. Theorem 1). Using Lemma 1, it suffices to show that there exists some $\delta>0$ such that $f_{\delta}^{\prime}(\log K+)>0$. We argue by contradiction, so, again using Lemma 1, suppose that $f_{\delta}^{\prime}(\log K+)<0$ for all $\delta>0$. This implies that, for each $\delta>0$, there exists some $\varepsilon>0$ such that $f_{\delta}(x)<f_{\delta}(\log K)=\delta$ for all $x \in(\log K, \log K+\varepsilon]$. Since $V \leq f_{\delta}$ (Lemma 1$)$, we deduce that $V(x)<\delta=\left(K-\mathrm{e}^{x}\right)^{+}+\delta$ for all $x \in(\log K, \log K+\varepsilon)$; hence, $y^{*}=\log K$ and, in fact, $V=f_{\delta}$ (by (8)).

Substituting $\tau_{\log K / 2}^{-}$into the right-hand side of (8) yields

$$
f_{\delta}(x) \geq \frac{K}{2} \mathrm{E}_{x}\left[\mathrm{e}^{-q \tau_{\log K / 2}^{-}} \mathbf{1}_{\left\{\tau_{\log K / 2}^{-}<T_{K}\right\}}\right] .
$$

This lower bound is strictly positive for $x>\log K$ since $\Pi \neq 0$ and does not depend on $\delta$. Hence, for small enough $\delta$, we deduce the existence of some $x>\log K$ such that $f_{\delta}(x)>\delta$, contradicting the fact that $f_{\delta}(x)=V(x) \leq \delta$ on $[\log K, \infty)$.

We now consider the optimal stopping time of the minimiser. For $\delta>\delta_{0}$, the same reasoning as above yields $y^{*}=\log K$.

For the case in which $\delta=\delta_{0}$, we note that, for any fixed $x$, the function $f_{\delta}(x)$ is continuous in $\delta$, as is easily seen from (8). Hence,

$$
f_{\delta_{0}}(x)=\lim _{\delta \downarrow \delta_{0}} f_{\delta}(x) \leq\left(K-\mathrm{e}^{x}\right)^{+}+\delta_{0},
$$

from which we can deduce that we still have $y^{*}=\log K$. Finally, let $\delta<\delta_{0}$. Again, much as above, we have $f_{\delta}^{\prime}(\log K+)>0$ and, thus, there exists $x>\log K$ for which $f_{\delta}(x)>\delta=$ $\left(K-\mathrm{e}^{x}\right)^{+}+\delta$. Since, trivially, $V$ is bounded above by this upper payoff function, it cannot be true that $f_{\delta}=V$ and, thus, it can also not be true that $y^{*}=\log K$, so we indeed arrive at $y^{*}>\log K$.

Remark 2. From the proof of Theorem 4 we see that this result is essentially due to the upper payoff function $\left(K-\mathrm{e}^{x}\right)^{+}+\delta$ having a kink at the point where it first touches the value function as $\delta$ decreases (namely, $\log K$ ). That is, if we only slightly alter the upper payoff function in an environment of $\log K$ so that it has a continuous derivative, we would expect the optimal stopping time for the minimiser to be $T_{\left[y_{1}^{*}, y_{2}^{*}\right]}$ with $y_{1}^{*}<\log K<y_{2}^{*}$ for all $\delta \in(0, \bar{\delta})$ and any spectrally negative Lévy process $X$.

Next we provide expressions that complement those of Theorem 2. Recall that (7) provides us with a formula for $V$ on $(-\infty, \log K]$, so we can make use of the following function:

$$
w_{\delta}(x)= \begin{cases}V(x) & \text { for } x<\log K, \\ \delta & \text { for } x \geq \log K\end{cases}
$$

Theorem 5. Suppose that $\Pi \neq 0$. Then the following assertions hold.

(i) Suppose that $\sigma_{X}>0$. Then $\delta_{0}$ is the unique solution on $(0, \bar{\delta})$ to the equation in $\delta$ :

$$
\int_{t<0} \int_{u<t}\left(w_{\delta}(t+\log K)-\delta\right) \mathrm{e}^{-\Phi(q)(t-u)} \Pi(\mathrm{d} u) \mathrm{d} t=\frac{\delta q}{\Phi(q)} .
$$


(ii) Suppose that $y^{*}>\log K$ (i.e. $\sigma_{X}>0$ and $\delta<\delta_{0}$, or $\sigma_{X}=0$ and $\delta<\bar{\delta}$ ). Then $y^{*}$ is the unique solution on $(\log K, \infty)$ to the equation in $y$ :

$$
\int_{t<0} \int_{u<t}\left(w_{\delta}(t+y)-\delta\right) \mathrm{e}^{-\Phi(q)(t-u)} \Pi(\mathrm{d} u) \mathrm{d} t=\frac{\delta q}{\Phi(q)} .
$$

Furthermore, $V(x)=\delta$ for $x \in\left[\log K, y^{*}\right]$ and, for $x \in\left(y^{*}, \infty\right)$,

$$
V(x)=\delta Z^{(q)}\left(x-y^{*}\right)-\int_{t<0} \int_{u<t}\left(w_{\delta}\left(t+y^{*}\right)-\delta\right) W^{(q)}\left(x-y^{*}-t+u\right) \Pi(\mathrm{d} u) \mathrm{d} t .
$$

Proof. First we introduce the function

$$
h(x, y):=\mathrm{E}_{x}\left[\mathrm{e}^{-q \tau_{y}^{-}} w_{\delta}\left(X_{\tau_{y}^{-}}\right)\right]
$$

for $x>y \geq \log K$. Observe that, by the lack of positive jumps, $h(\cdot, y)$ is the optimal value the maximiser can obtain when the minimiser chooses the stopping region $[\log K, y]$. Hence, in particular, $V(x)=h\left(x, y^{*}\right)$.

Denote by $u^{(q)}(s, t)$ the resolvent density of $X$ started at $s>0$ and killed at the first passage below 0 . Invoking the compensation formula (see, e.g. Theorem 4.4 of [16]) leads to

$$
\begin{aligned}
h(x, y) & =\delta \mathrm{E}_{x}\left[\mathrm{e}^{-q \tau_{y}^{-}}\right]+\mathrm{E}_{x}\left[\mathrm{e}^{-q \tau_{y}^{-}}\left(w_{\delta}\left(X_{\tau_{y}^{-}}\right)-\delta\right) \mathbf{1}_{\left\{X_{\tau_{y}^{-}}<\log K\right\}}\right] \\
& =\delta \mathrm{E}_{x}\left[\mathrm{e}^{-q \tau_{y}^{-}}\right]+\int_{t<\log K-y} \int_{u<t}\left(w_{\delta}(t+y)-\delta\right) u^{(q)}(x-y, t-u) \Pi(\mathrm{d} u) \mathrm{d} t \\
& =\delta \mathrm{E}_{x}\left[\mathrm{e}^{-q \tau_{y}^{-}}\right]+\int_{t<0} \int_{u<t}\left(w_{\delta}(t+y)-\delta\right) u^{(q)}(x-y, t-u) \Pi(\mathrm{d} u) \mathrm{d} t,
\end{aligned}
$$

where the final equality is due to the fact that $w_{\delta}=\delta$ on $[\log K, y]$. We know that (see, e.g. Theorem 8.1 and Corollary 8.8 of [16], respectively)

$$
\mathrm{E}_{x}\left[\mathrm{e}^{-q \tau_{y}^{-}}\right]=Z^{(q)}(x-y)-\frac{q}{\Phi(q)} W^{(q)}(x-y)
$$

and

$$
u^{(q)}(s, t)=\mathrm{e}^{-\Phi(q) t} W^{(q)}(s)-W^{(q)}(s-t)
$$

hence,

$$
\begin{aligned}
h(x, y)= & \int_{t<0} \int_{u<t}\left(w_{\delta}(t+y)-\delta\right)\left(\mathrm{e}^{-\Phi(q)(t-u)} W^{(q)}(x-y)\right. \\
& \left.-W^{(q)}(x-y-t+u)\right) \Pi(\mathrm{d} u) \mathrm{d} t \\
& +\delta\left(Z^{(q)}(x-y)-\frac{q}{\Phi(q)} W^{(q)}(x-y)\right) .
\end{aligned}
$$

Furthermore, when $X$ is of unbounded variation, we can compute, for $x>y$,

$$
\begin{aligned}
\frac{\partial}{\partial x} h(x, y)= & \delta\left(q W^{(q)}(x-y)-\frac{q}{\Phi(q)} W^{(q)^{\prime}}(x-y)\right) \\
& +\int_{t<0} \int_{u<t}\left(w_{\delta}(t+y)-\delta\right)\left(\mathrm{e}^{-\Phi(q)(t-u)} W^{(q)^{\prime}}(x-y)\right. \\
& \left.\quad-W^{(q)^{\prime}}(x-y-t+u)\right) \Pi(\mathrm{d} u) \mathrm{d} t,
\end{aligned}
$$


and we can let $x \downarrow y$ to arrive at

$$
\frac{\partial}{\partial x} h(y+, y)=\left(\int_{t<0} \int_{u<t}\left(w_{\delta}(t+y)-\delta\right) \mathrm{e}^{-\Phi(q)(t-u)} \Pi(\mathrm{d} u) \mathrm{d} t-\frac{q \delta}{\Phi(q)}\right) W^{(q)^{\prime}}(0+) .
$$

(i) Recall the function $f_{\delta}$ as defined in (8), and recall, in particular, from the proof of Lemma 1 that $\delta_{0}$ is the unique $\delta \in(0, \bar{\delta})$ for which $f_{\delta}^{\prime}(\log K+)=0$. Furthermore, note that $f_{\delta}(x)=h(x, \log K)$ for $x>\log K$, since both sides equal the optimal value the maximiser can obtain when the minimiser only stops when $X$ hits $\log K$. Combining these observations with (14) and $W^{(q)^{\prime}}(0+)=2 / \sigma_{X}^{2} \neq 0$ yields the result.

(ii) We first consider the case when $X$ is of bounded variation. We know from Theorem 4 of BK that we have a continuous fit, i.e. $V\left(y^{*}+\right)=\delta$. Since the integrand in (13) is bounded and equal to 0 for $t<\log K-y$, we can take the limit inside the integrals to deduce that

$$
h(y+, y)=\delta-\frac{q \delta}{d \Phi(q)}+\frac{1}{d} \int_{t<0} \int_{u<t}\left(w_{\delta}(t+y)-\delta\right) \mathrm{e}^{-\Phi(q)(t-u)} \Pi(\mathrm{d} u) \mathrm{d} t,
$$

where $d=\left(W^{(q)}(0)\right)^{-1}$ denotes the drift of $X$, and so, using $V\left(y^{*}+\right)=h\left(y^{*}+, y^{*}\right)$, it follows that $y^{*}$ indeed solves (11). For uniqueness, the function $w_{\delta}=V$ is strictly decreasing on $(-\infty, \log K]$ and $\delta=V\left(y^{*}\right)=h\left(y^{*}+, y^{*}\right)$. Since $q>0$, the minimiser would not stop at points in $[\log K, \infty]$ from which the process cannot jump into $(-\infty, \log K)$ and, thus, $\log K-y^{*}>l:=\sup \{x: \Pi(-\infty, x)=0\}$. Combining these observations we can imply that $h(y+, y)$ is a strictly decreasing function on $[\log K, \log K-l]$.

Next consider the case when $X$ is of unbounded variation. Now Theorem 4 of BK tells us that we have a smooth fit, i.e. $V^{\prime}\left(y^{*}+\right)=0$. Using $V(x)=h\left(x, y^{*}\right)$ together with (14), again yields the fact that $y^{*}$ solves (11), and uniqueness follows in the same way as in the previous paragraph.

Finally, (12) is readily seen from $V(x)=h\left(x, y^{*}\right),(13)$, and the fact that $y^{*}$ satisfies (11).

We conclude this section with some properties of $y^{*}$ as a function of $\delta$. Note that, by spectral negativity, $\Pi \neq 0$ implies that $\sup \{x: \Pi(-\infty, x)=0\}<0$.

Theorem 6. Suppose that $\Pi \neq 0$. Then $y^{*}(\delta)$ is continuous and decreasing as a function of $\delta$, with $y^{*}(\bar{\delta}-)=\log K$ if $\sigma_{X}=0\left(y^{*}\left(\delta_{0}-\right)=\log K\right.$ if $\left.\sigma_{X}>0\right)$ and $y^{*}(0+)=\log K-$ $\sup \{x: \Pi(-\infty, x)=0\}$.

Proof. We write $V_{\delta}$ to stress the dependence of the value function on $\delta$. Continuity of $y^{*}(\delta)$ is clear, as Theorem 5(ii) and the fact that $w_{\delta}$ is continuous in $\delta$ (see the argument for continuity of $\delta \mapsto V_{\delta}$ below) allow us to apply the implicit function theorem.

To see that it is decreasing, it suffices to show that $\delta \mapsto V_{\delta}(x)-\delta$ is decreasing. For this, take $\delta_{1}<\delta_{2}$ and let $\left(\tau_{1}^{*}, \sigma_{1}^{*}\right)$ denote the saddle point when $\delta=\delta_{1}$. Then $V_{\delta_{1}}$ is the value when the supremum over all $\tau$ is taken in the expected payoff corresponding to the pair $\left(\tau, \sigma_{1}^{*}\right)$. As $\sigma_{1}^{*}$ is also feasible for the minimiser when $\delta=\delta_{2}, V_{\delta_{2}}$ is bounded above by the value when the supremum over all $\tau$ is taken in the expected payoff corresponding to the pair $\left(\tau, \sigma_{1}^{*}\right)$. This yields

$$
\begin{aligned}
V_{\delta_{2}}(x)-V_{\delta_{1}}(x) \leq \sup _{\tau} \mathrm{E}_{x}\left[\mathrm{e}^{-q \sigma_{1}^{*}}\left(\left(K-\mathrm{e}^{X_{\sigma_{1}^{*}}}\right)^{+}+\delta_{2}\right) \mathbf{1}_{\left\{\sigma_{1}^{*}<\tau\right\}}\right. \\
\left.\quad-\mathrm{e}^{-q \sigma_{1}^{*}}\left(\left(K-\mathrm{e}^{X_{\sigma_{1}^{*}}}\right)^{+}+\delta_{1}\right) \mathbf{1}_{\left\{\sigma_{1}^{*}<\tau\right\}}\right] \\
\leq \delta_{2}-\delta_{1},
\end{aligned}
$$

as required. 
Next, by the monotonicity, the limits mentioned in the theorem exist. First we show that $y^{*}(0+)=\log K-l$, where $l:=\sup \{x: \Pi(-\infty, x)=0\}$. Suppose that we have $y^{*}(0+)<\log K-l$. Then, for some $x_{1} \in\left(y^{*}(0+), \log K-l\right)$ and any $\delta>0$, we have $\mathrm{P}_{x_{1}}\left(\tau_{\log K / 2}^{-}<T_{\left[\log K, y^{*}(\delta)\right]}\right) \geq \mathrm{P}_{x_{1}}\left(\tau_{\log K / 2}^{-}<T_{\left[\log K, y^{*}(0+)\right]}\right)>0$. So, starting from $x_{1}$, if the maximiser chooses $\tau_{\log K / 2}^{-}$, he ensures a strictly positive value, independent of $\delta$. But this of course contradicts $V_{\delta}\left(x_{1}\right) \leq \delta \downarrow 0$ as $\delta \downarrow 0$. If we have $y^{*}(0+)>\log K-l$ then, for some $x_{2} \in\left(\log K-l, y^{*}(0+)\right)$, we have, for small enough $\delta, x_{2} \leq y^{*}(\delta)$ and, consequently, $V_{\delta}\left(x_{2}\right)=\delta$. But the minimiser can do better, that is, in fact, we have $V_{\delta}\left(x_{2}\right)<\delta$, as is easily seen. Namely, the minimiser can choose $T_{[\log K, \log K-l]}$, so that starting from $x_{2}>\log K-l$ the maximiser can at most get discounted $\delta$, the discount factor being strictly less than 1 since $q>0$ and $X$ is right continuous.

Next suppose that $\sigma_{X}>0$. We show that $y^{*}\left(\delta_{0}-\right)=\log K$. Suppose that we have $y^{*}\left(\delta_{0}-\right)>\log K$. Note that, for any $x, \delta \mapsto V_{\delta}(x)$ is continuous, since, for $\delta_{1}<\delta_{2}$, trivially, $V_{\delta_{2}}(x) \geq V_{\delta_{1}}(x)$ and (15) holds. So, for $\log K<x_{1}<x_{2}<y^{*}\left(\delta_{0}-\right)$, it follows that $V_{\delta}\left(x_{1}\right)-V_{\delta}\left(x_{2}\right) \rightarrow V_{\delta_{0}}\left(x_{1}\right)-V_{\delta_{0}}\left(x_{2}\right)=\delta_{0}-\delta_{0}=0$ as $\delta \downarrow \delta_{0}$. But the difference $V_{\delta}\left(x_{1}\right)-$ $V_{\delta}\left(x_{2}\right)$ does not vanish as $\delta \downarrow \delta_{0}$, as follows easily from the homogeneity of $X$. More precisely, denoting by $\left(\tau_{1}^{*}, \sigma_{1}^{*}\right)$ and $\left(\tau_{2}^{*}, \sigma_{2}^{*}\right)$ the saddle point when starting from $x_{1}$ and $x_{2}$, respectively, similar arguments as those leading to (15) yield in this case

$$
V_{\delta}\left(x_{1}\right) \geq \mathrm{E}\left[\mathrm{e}^{-q \tau_{2}^{*}}\left(K-\mathrm{e}^{x_{1}+X_{\tau_{2}^{*}}}\right)^{+} \mathbf{1}_{\left\{\tau_{2}^{*} \leq \sigma_{1}^{*}\right\}}+\mathrm{e}^{-q \sigma_{1}^{*}}\left(\left(K-\mathrm{e}^{x_{1}+X_{\sigma_{1}^{*}}}\right)^{+}+\delta\right) \mathbf{1}_{\left\{\sigma_{1}^{*}<\tau_{2}^{*}\right\}}\right]
$$

and

$$
V_{\delta}\left(x_{2}\right) \leq \mathrm{E}\left[\mathrm{e}^{-q \tau_{2}^{*}}\left(K-\mathrm{e}^{x_{2}+X_{\tau_{2}^{*}}}\right)^{+} \mathbf{1}_{\left\{\tau_{2}^{*} \leq \sigma_{1}^{*}\right\}}+\mathrm{e}^{-q \sigma_{1}^{*}}\left(\left(K-\mathrm{e}^{x_{2}+X_{\sigma_{1}^{*}}}\right)^{+}+\delta\right) \mathbf{1}_{\left\{\sigma_{1}^{*}<\tau_{2}^{*}\right\}}\right]
$$

thus,

$$
V_{\delta}\left(x_{1}\right)-V_{\delta}\left(x_{2}\right) \geq \mathrm{E}\left[\mathrm{e}^{-q \kappa}\left(\left(K-\mathrm{e}^{x_{1}+X_{\kappa}}\right)^{+}-\left(K-\mathrm{e}^{x_{2}+X_{\kappa}}\right)^{+}\right)\right],
$$

where $\kappa=\sigma_{1}^{*} \wedge \tau_{2}^{*}=\inf \left\{t>0 \mid X_{t}=\log K-x_{1}\right\} \wedge \inf \left\{t>0 \mid X_{t}<x^{*}(\delta)-x_{2}\right\}$. Clearly, since $x^{*}(\delta) \leq \log K$ and $x_{1}<x_{2}$, the right-hand side of (16) is strictly positive if and only if $\mathrm{P}\left(\tau_{2}^{*}<\sigma_{1}^{*}\right)>0$. Even after taking the limit for $\delta \downarrow \delta_{0}$ this probability is positive on account of $\Pi \neq 0$.

Finally, $y^{*}(\bar{\delta}-)=\log K$ when $\sigma_{X}=0$ can be shown by the same arguments, taking into account here that we have $\sigma^{*}=\infty$ for $\delta>\bar{\delta}$.

\section{Jump diffusion case}

In this section we translate the general results from Section 2 to the particular case of a jump diffusion with downwards directed, exponentially distributed jumps. In this case, which is quite popular in practical applications in finance for example, owing to its tractable nature, the expressions become much more explicit. In particular, a formula exists that explicitly expresses $y^{*}$ in terms of $x^{*}$; cf. Proposition 3(iv).

In the sequel we set

$$
X_{t}=\sigma_{X} W_{t}+\mu t-\sum_{i=1}^{N_{t}} \xi_{i}, \quad t \geq 0,
$$

where $\sigma_{X}>0, \mu \in \mathbb{R}, N$ is a Poisson process with intensity $\lambda>0$ counting the jumps, and $\left(\xi_{i}\right)_{i \geq 0}$ is an independent and identically distributed sequence of random variables following an exponential distribution with parameter $\theta>0$. 
Proposition 1 below gives formulae for the scale functions in this jump diffusion case (recall $\mathrm{P}^{c}$ as defined in (5)).

Proposition 1. Let $c, r \geq 0$. The following assertions hold for $X$ given by (17) under $\mathrm{P}^{c}$.

(i) The Laplacian is given by

$$
\psi_{c}(z)=\psi(z+c)-\psi(c)=\frac{\sigma_{X}^{2}}{2} z^{2}+\left(\sigma_{X}^{2} c+\mu\right) z-\frac{\lambda \theta z}{(\theta+z+c)(\theta+c)} .
$$

The function $z \mapsto \psi_{c}(z)-r$ has three $0 s, \beta_{1}(c, r)<-\theta-c<\beta_{2}(c, r) \leq \beta_{3}(c, r)$, with $\beta_{2}(c, r)<0<\beta_{3}(c, r)$ if $r>0, \beta_{2}(c, r)=0<\beta_{3}(c, r)$ if $r=0$ and $\psi_{c}^{\prime}(0) \leq 0$, and $\beta_{2}(c, r)<0=\beta_{3}(c, r)$ if $r=0$ and $\psi_{c}^{\prime}(0) \geq 0$.

(ii) In particular, if $r=\psi(1)>0$, we have

$$
\beta_{1,2}(0, r)=-\left(\frac{\theta}{2}+\frac{r}{\sigma_{X}^{2}}+\frac{\lambda}{\sigma_{X}^{2}(\theta+1)}\right) \pm \sqrt{\left(\frac{\theta}{2}+\frac{r}{\sigma_{X}^{2}}+\frac{\lambda}{\sigma_{X}^{2}(\theta+1)}\right)^{2}-\frac{2 r \theta}{\sigma_{X}^{2}}}
$$

and $\beta_{3}(0, r)=1$.

Define, for $i=1,2,3$, the constants

$$
C_{i}(c, r)=\frac{2\left(\theta+c+\beta_{i}(c, r)\right)}{\sigma_{X}^{2} \prod_{j \neq i}\left(\beta_{j}(c, r)-\beta_{i}(c, r)\right)} .
$$

We have the following formulae for the scale functions $W_{c}^{(r)}$ and $Z_{c}^{(r)}$ on $[0, \infty)$.

(iii) If $\beta_{2}(c, r) \neq 0$ or $\beta_{3}(c, r) \neq 0$, we have

$$
W_{c}^{(r)}(x)=\sum_{i=1}^{3} C_{i}(c, r) \mathrm{e}^{\beta_{i}(c, r) x},
$$

otherwise (necessarily $r=0$ ) we have

$$
W_{c}^{(0)}(x)=\frac{2}{\sigma_{X}^{2} \beta_{1}(c, 0)}\left((1-c-\theta) \mathrm{e}^{\beta_{1}(c, 0) x}-(\theta+c) x+\theta+c-1\right) .
$$

(i) If $r>0$, we have

$$
Z_{c}^{(r)}(x)=r \sum_{i=1}^{3} \frac{C_{i}(c, r)}{\beta_{i}(c, r)} \mathrm{e}^{\beta_{i}(c, r) x}
$$

while $Z_{c}^{(0)}(x)=1$.

Proof. The proof follows from definitions (2) and (3) by some elementary calculations. Also, see, e.g. [1].

In Propositions 2 and 3 below we assume for simplicity that $q>0$ and $q=\psi(1)$, i.e. we set $\mu:=q-\sigma_{X}^{2} / 2+\lambda /(\theta+1)$. (Note that condition (1) is met). This means that $\mathrm{P}$ is a so-called risk neutral measure in the sense that the discounted price process $\left(\mathrm{e}^{X_{t}-q t}\right)_{t \geq 0}$ is a P-martingale, as required in a financial modelling context. (However, the reader should have no difficulties translating the upcoming formulae to the situation for any $q \in[0, \psi(1)]$ if required.) Note that Proposition 1(ii) gives explicit formulae for the roots $\beta_{i}(0, q)$ in this case.

First we turn to formulae for the McKean optimal stopping problem (cf. Theorem 1). 
Proposition 2. The value function $U$ of the McKean optimal stopping problem is given by

$$
U(x)= \begin{cases}K-\mathrm{e}^{x} & \text { if } x \leq k^{*}, \\ c_{1} \mathrm{e}^{\beta_{1}(0, q)\left(x-k^{*}\right)}+c_{2} \mathrm{e}^{\beta_{2}(0, q)\left(x-k^{*}\right)} & \text { if } x>k^{*},\end{cases}
$$

where

$$
\begin{gathered}
c_{1}=\frac{\beta_{2}(0, q) K+\left(1-\beta_{2}(0, q)\right) \mathrm{e}^{k^{*}}}{\beta_{2}(0, q)-\beta_{1}(0, q)}, \quad c_{2}=\frac{\beta_{1}(0, q) K+\left(1-\beta_{1}(0, q)\right) \mathrm{e}^{k^{*}}}{\beta_{1}(0, q)-\beta_{2}(0, q)} \\
\mathrm{e}^{k^{*}}=\frac{K q}{\sigma_{X}^{2} / 2+q+\lambda /(\theta+1)^{2}} .
\end{gathered}
$$

Proof. Direct derivations of these formulae can be found in [14] for example. Alternatively, substituting the formulae of Proposition 1 into the results of Theorem 1, we see that we can write

$$
U(x)=K q \sum_{i=1}^{3} \frac{C_{i}(0, q)}{\beta_{i}(0, q)} \mathrm{e}^{\beta_{i}(0, q)\left(x-k^{*}\right)}-\mathrm{e}^{x} \quad \text { and } \quad \mathrm{e}^{k^{*}}=K \frac{\psi(1)}{\psi^{\prime}(1)} .
$$

Applying the identity

$$
\frac{\sigma_{X}^{2}}{2} \prod_{i=1}^{3}\left(z-\beta_{i}(c, q)\right)=(\theta+z+c)\left(\psi_{c}(z)-q\right) \text { for } z \neq-\theta-c
$$

to this particular case (i.e. $c=0, q=\psi(1)$, and $\beta_{3}(0, q)=1$ ), dividing both sides by $z-1$, and taking the limit for $z \rightarrow 1$, we obtain

$$
\sigma_{X}^{2}\left(1-\beta_{1}(0, q)\right)\left(1-\beta_{2}(0, q)\right)=2(\theta+1) \psi^{\prime}(1) .
$$

Substituting this into the equation for $\mathrm{e}^{k^{*}}$ we obtain

$$
\mathrm{e}^{k^{*}}=\frac{2(\theta+1) K q}{\sigma_{X}^{2}\left(\beta_{2}(0, q)-1\right)\left(\beta_{1}(0, q)-1\right)} .
$$

Using this expression in (18), together with $\beta_{1}(0, q) \beta_{2}(0, q)=2 q \theta / \sigma_{X}^{2}$ (from (19) with $z=0$ ), the stated formula for $U$ indeed follows.

Now we are ready to consider the formulae for the optimal exercise levels $x^{*}$ and $y^{*}$, and the value function $V$ of the McKean game. Recall that, for $\delta \geq U(\log K)$, the game degenerates to the McKean optimal stopping problem.

Proposition 3. Consider the McKean game driven by (17). Recall that $\bar{\delta}=U(\log K)$. We assume throughout that $\delta<\bar{\delta}$.

(i) The optimal level $x^{*}=x^{*}(\delta)$ is the unique solution to the equation in $x$ :

$$
q \sum_{i=1}^{3} \frac{C_{i}(0, q)}{\beta_{i}(0, q)} K^{\beta_{i}(0, q)} \mathrm{e}^{-\beta_{i}(0, q) x}-1=\frac{\delta}{K} .
$$

On $\left(-\infty, x^{*}\right]$ we have $V(x)=K-\mathrm{e}^{x}$ and on $\left(x^{*}, \log K\right]$ we have

$$
V(x)=K q \sum_{i=1}^{3} \frac{C_{i}(0, q)}{\beta_{i}(0, q)} \mathrm{e}^{\beta_{i}(0, q)\left(x-x^{*}\right)}-\mathrm{e}^{x} .
$$


(ii) The threshold $\delta_{0} \in(0, \bar{\delta})$ is the unique solution to the equation in $z$ :

$$
q \sum_{i=1}^{3} \frac{C_{i}(0, q) K^{\beta_{i}(0, q)}}{\beta_{i}(0, q)\left(\theta+\beta_{i}(0, q)\right)} \mathrm{e}^{-\beta_{i}(0, q) x^{*}(z)}-\frac{\lambda+(\theta+1) q}{\lambda \theta K} z=\frac{1}{\theta+1} .
$$

(iii) Suppose that $\delta \in\left[\delta_{0}, \bar{\delta}\right)$. We have $y^{*}=\log K$ and, on $[\log K, \infty)$,

$$
V(x)=K \sum_{i=1}^{2} C_{i}(0, q)\left(\frac{q \mathrm{e}^{-\beta_{i}(0, q) x^{*}}}{\beta_{i}(0, q)}+K^{-\beta_{i}(0, q)}\left(\psi^{\prime}(1)-K q \mathrm{e}^{-x^{*}}\right)\right) \mathrm{e}^{\beta_{i}(0, q) x} .
$$

(iv) Suppose that $\delta \in\left(0, \delta_{0}\right)$. We have

$$
\mathrm{e}^{\theta y^{*}}=\frac{\lambda \theta K^{\theta+1}}{(\theta+1) q \delta}\left(q \sum_{i=1}^{3} \frac{C_{i}(0, q) K^{\beta_{i}(0, q)}}{\beta_{i}(0, q)\left(\theta+\beta_{i}(0, q)\right)} \mathrm{e}^{-\beta_{i}(0, q) x^{*}}-\frac{1}{\theta+1}-\frac{\delta}{\theta K}\right) .
$$

On $\left[\log K, y^{*}\right]$ we have $V(x)=\delta$ and on $\left(y^{*}, \infty\right)$ we have

$$
V(x)=\frac{\delta}{\beta_{2}(0, q)-\beta_{1}(0, q)}\left(\beta_{2}(0, q) \mathrm{e}^{\beta_{1}(0, q)\left(x-y^{*}\right)}-\beta_{1}(0, q) \mathrm{e}^{\beta_{2}(0, q)\left(x-y^{*}\right)}\right) .
$$

Proof. (i) Apply Proposition 1 to the formulae of Theorem 2(ii).

(ii) Apply Proposition 1 to Theorem 5(i).

(iii) Apply Proposition 1 to the formula of Theorem 2(ii) to obtain

$$
V(x)=K \sum_{i=1}^{3} C_{i}(0, q)\left(\frac{q \mathrm{e}^{-\beta_{i}(0, q) x^{*}}}{\beta_{i}(0, q)}+K^{-\beta_{i}(0, q)}\left(\psi^{\prime}(1)-K q \mathrm{e}^{-x^{*}}\right)\right) \mathrm{e}^{\beta_{i}(0, q) x}-\mathrm{e}^{x}
$$

and use (20) to see that the terms involving the exponential of a positive factor times $x$ vanish. (Of course, we can also reason directly that they should cancel, since otherwise $V$ would not stay bounded for large $x$, which it should by definition.)

(iv) For $y^{*}$, apply Proposition 1 to Theorem 5(ii) and simplify to arrive at the stated formula. Note that

$$
\sum_{i=1}^{3} \frac{C_{i}(0, q)}{\beta_{i}(0, q)\left(\theta+\beta_{i}(0, q)\right)}=\frac{2}{\sigma_{X}^{2} \prod_{i=1}^{3} \beta_{i}(0, q)}=\frac{1}{\theta q}
$$

where the final equality follows by (19).

For $V$, apply Proposition 1 to Theorem 5(ii) and simplify, making use of the formula for $y^{*}$ and, in particular, Proposition 1(ii).

We conclude with some plots in this jump diffusion setting to illustrate the main result from this paper.

Figures 1 and 2 show the value function $V$ in the two different cases $\delta \in\left[\delta_{0}, \bar{\delta}\right)$ and $\delta \in\left(0, \delta_{0}\right)$.

Figure 3 shows a plot of $\bar{\delta}$ and $\delta_{0}$ as a function of $\sigma_{X}$. (Note that this really means only $\sigma_{X}$ changes; hence, the equation $q=\psi(1)$ does not (necessarily) hold as $\psi$ changes with $\sigma_{X}$.) This figure can be explained as follows. If $\sigma_{X} \downarrow 0, U$ converges to the value of the McKean optimal stopping problem for $X$ with $\sigma_{X}=0$ and, hence, $\bar{\delta}=U(\log K)$ also has some limit in the interval $(0, K)$. The figure suggests that the difference between $\bar{\delta}$ and $\delta_{0}$ vanishes as $\sigma_{X} \downarrow 0$, which might be explained as follows. As pointed out in Remark $1, \delta \in\left(\delta_{0}, \bar{\delta}\right)$ means that, when $X$ starts above $\log K$, the probability of hitting $\log K$ before it reaches levels (far) below $\log K$ 


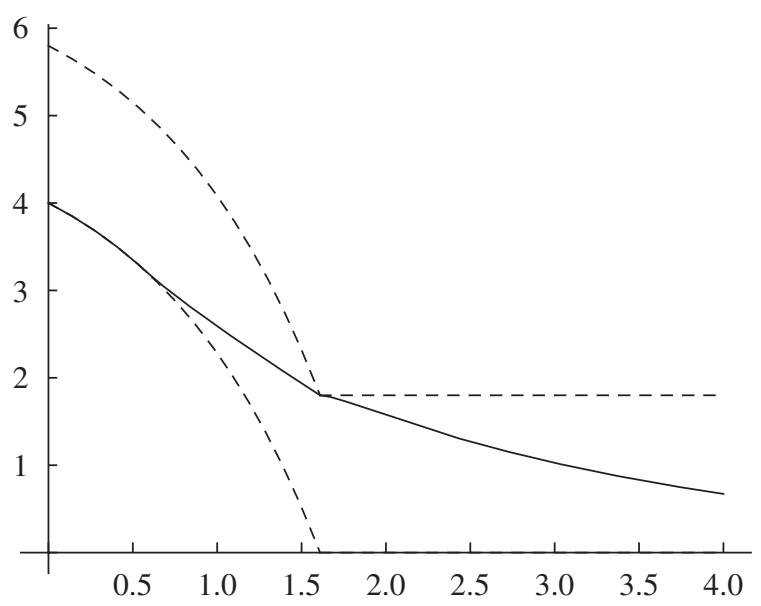

FIGURE 1: A plot of the value function $V$ for the case in which $\delta \in\left[\delta_{0}, \bar{\delta}\right)$, so $y^{*}=\log K$. The dashed lines are the upper and lower payoff functions, the solid line is $V$. Here $K=5, \delta=1.8, \bar{\delta}=2.08$, $\delta_{0}=1.76$, and $x^{*}=0.58$.

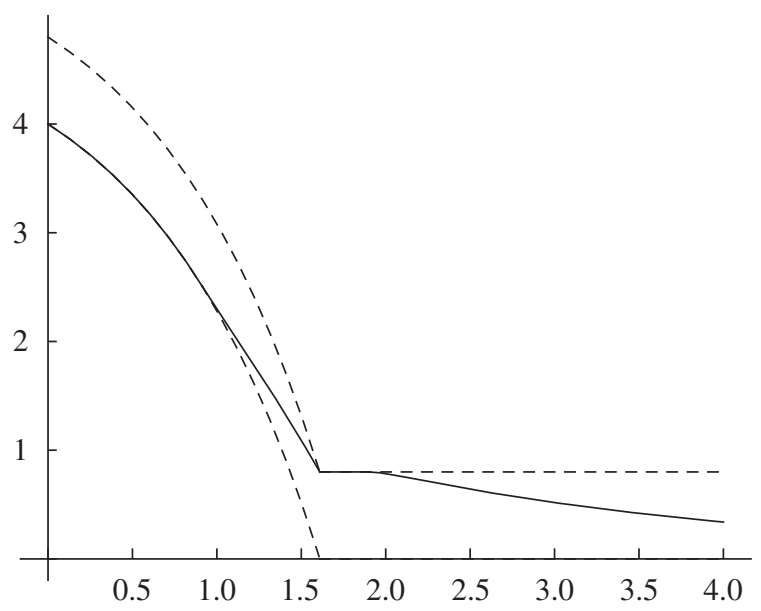

Figure 2: A plot of the value function $V$ for the case in which $\delta \in\left(0, \delta_{0}\right)$, so $y^{*}>\log K$. The dashed lines are the upper and lower payoff functions, the solid line is the value function $V$. Here $K, \delta_{0}$, and $\bar{\delta}$ are as in Figure $1, \delta=0.8, x^{*}=0.91$, and $y^{*}=1.90$.

is large enough to have $y^{*}=\log K$. Obviously, this probability vanishes together with $\sigma_{X}$, and, hence, the length of the interval $\left(\delta_{0}, \bar{\delta}\right)$ vanishes as $\sigma_{X} \downarrow 0$. Furthermore, as $\sigma_{X} \rightarrow \infty$, for the maximiser in the McKean optimal stopping problem, the negative effect of discounting vanishes in the sense that the first entry time of any interval has a density approaching the Dirac measure in 0 . Hence, $k^{*} \rightarrow-\infty$ and $U(x) \uparrow K$ for any $x$. In particular, $\bar{\delta}=U(\log K) \uparrow K$ also. The vanishing of $\bar{\delta}$ as $\sigma_{X} \rightarrow \infty$ is explained as above by the fact that increasing $\sigma_{X}$ means that the probability of hitting $\log K$ before $X$ falls (far) below $\log K$ increases, and, hence, the 


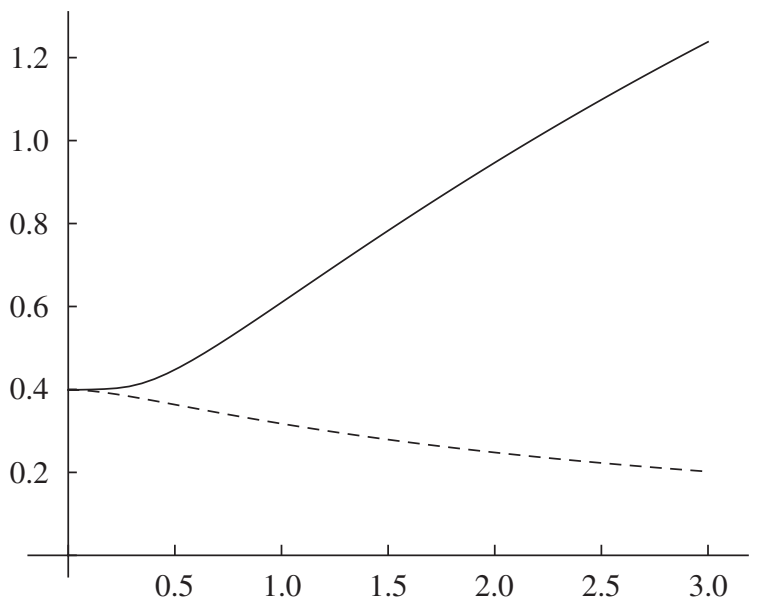

FIGURE 3: A plot of $\bar{\delta}$ (solid line) and $\delta_{0}$ (dashed line) as a function of $\sigma_{X}$.

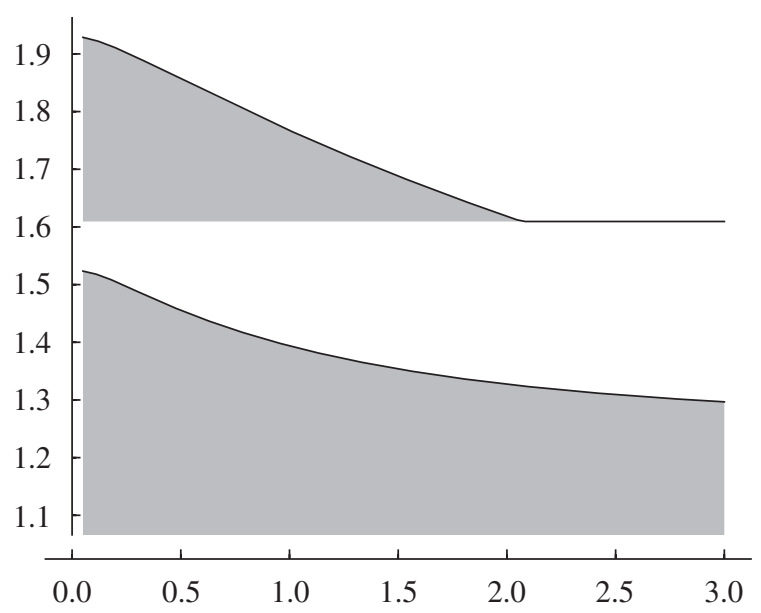

Figure 4: A graphical illustration of the optimal stopping regions $\left(-\infty, x^{*}\right]$ (maximizer) and $\left[\log K, y^{*}\right]$ (minimizer) as a function of $\sigma_{X}$. The upper solid line is $y^{*}\left(\sigma_{X}\right)$, the lower solid line is $x^{*}\left(\sigma_{X}\right)$.

effect of the negative jumps is of vanishing relevance. In the limit therefore the minimiser does not need to choose a $y^{*}>\log K$ for any $\delta>0$.

Finally, Figure 4 shows how $x^{*}$ and $y^{*}$ vary with $\sigma_{X}$. Note that the behaviour of $y^{*}$ is indeed consistent with the structure Figure 3 suggests.

\section{References}

[1] Avram, F., Kyprianou, A. E. And Pistorius, M. R. (2004). Exit problems for spectrally negative Lévy processes and applications to (Canadized) Russian options. Ann. Appl. Prob. 14, 215-238.

[2] Baurdoux, E. J. and Kyprianou, A. E. (2008). The McKean stochastic game driven by a spectrally negative Lévy process. Electron J. Prob. 8, 173-197. 
[3] Baurdoux, E. J. And Kyprianou, A. E. (2009). The Shepp-Shiryaev stochastic game driven by a spectrally negative Lévy process. Theory Prob. Appl. 53, 481-499.

[4] Baurdoux, E. J., Kyprianou, A. E. and Pardo, J. C. (2011). The Gapeev-Kühn stochastic game driven by a spectrally positive Lévy process. To appear in Stoch. Process. Appl.

[5] Bertoin, J. (1996). Lévy Processes. Cambridge University Press.

[6] Bichteler, K. (2002). Stochastic Integration with Jumps. Cambridge University Press.

[7] Chan, T. (2004). Some applications of Lévy processes in insurance and finance. Finance 25, 71-94.

[8] Doney, R. A. (2005). Some excursion calculations for spectrally one-sided Lévy processes. In Séminaire de Probabilités XXXVIII (Lecture Notes Math. 1857), Springer, Berlin, pp. 5-15.

[9] Dynkin, E. B. (1969). A game-theoretic version of an optimal stopping problem. Dokl. Akad. Nauk. SSSR 185, 16-19.

[10] Ekström, E. ANd Peskir, G. (2008). Optimal stopping games for Markov processes. SIAM J. Control Optimization 2, 684-702.

[11] Gapeev, P. V. and KüHn, C. (2005). Perpetual convertible bonds in jump-diffusion models. Statist. Decisions 23, 15-31.

[12] Kallsen, J. And Kühn, C. (2004). Pricing derivatives of American and game type in incomplete markets. Finance Stoch. 8, 261-284.

[13] KifER, Y. (2000). Game options. Finance Stoch. 4, 443-463.

[14] Kou, S. AND WANG, H. (2002). Option pricing under a jump-diffusion model. Manag. Sci. 50, 1178-1192.

[15] Kyprianou, A. E. (2004). Some calculations for Israeli options. Finance Stoch. 8, 73-86.

[16] Kyprianou, A. E. (2006). Introductory Lectures on Fluctuations of Lévy Processes with Applications. Springer, Berlin.

[17] McKeAn, H. (1965). Appendix: a free boundary problem for the heat equation arising from a problem of mathematical economics. Indust. Manag. Rev. 6, 32-39.

[18] MordecKI, E. (2002). Optimal stopping and perpetual options for Lévy processes. Finance Stoch. 6, $473-493$. 